\title{
Blow up for non-Newtonian equations with two nonlinear sources
}

\author{
Burhan Selcuk \\ Department of Computer Engineering, Karabuk University, Karabuk, Turkey
}

\begin{abstract}
This paper studies the following two non-Newtonian equations with nonlinear boundary conditions. Firstly, we show that finite time blow up occurs on the boundary and we get a blow up rate and an estimate for the blow up time of the equation $k_{t}=\left(\left|k_{x}\right|^{r-2} k_{x}\right)_{x}$, $(x, t) \in(0, L) \times(0, T)$ with $k_{x}(0, t)=k^{\alpha}(0, t), k_{x}(L, t)=k^{\beta}(L, t), t \in(0, T)$ and initial function $k(x, 0)=k_{0}(x), x \in[0, L]$ where $r \geq 2, \alpha, \beta$ and $L$ are positive constants. Secondly, we show that finite time blow up occurs on the boundary, and we get blow up rates and estimates for the blow up time of the equation $k_{t}=\left(\left|k_{x}\right|^{r-2} k_{x}\right)_{x}+k^{\alpha}$, $(x, t) \in(0, L) \times(0, T)$ with $k_{x}(0, t)=0, k_{x}(L, t)=k^{\beta}(L, t), t \in(0, T)$ and initial function $k(x, 0)=k_{0}(x), x \in[0, L]$ where $r \geq 2, \alpha, \beta$ and $L$ are positive constants.
\end{abstract}

Mathematics Subject Classification (2020). 35K20, 35K55, 35B44, 35B50

Keywords. heat equation, nonlinear parabolic equation, blow up, maximum principles

\section{Introduction}

The non-Newtonian equations appear in different branches of applied sciences where this simple model appears in a natural way. Some of them are population dynamics, chemical reactions, heat transfer. Non-Newtonian equations occupies an important place in applied mathematics literature. Many mathematicians have been interested in the solutions and properties of blow up problems with various boundary conditions for many years (see $[1,4,6,8-11])$.

In this paper, we consider the following two non-Newtonian equations with two nonlinear sources to obtain blow up properties extracted from the solutions:

$$
\left\{\begin{array}{l}
k_{t}=\left(\left|k_{x}\right|^{r-2} k_{x}\right)_{x}, \quad(x, t) \in(0, L) \times(0, T), \\
k_{x}(0, t)=k^{\alpha}(0, t), k_{x}(L, t)=k^{\beta}(L, t), t \in(0, T), \\
k(x, 0)=k_{0}(x), x \in[0, L],
\end{array}\right.
$$

and

$$
\left\{\begin{array}{l}
k_{t}=\left(\left|k_{x}\right|^{r-2} k_{x}\right)_{x}+k^{\alpha},(x, t) \in(0, L) \times(0, T), \\
k_{x}(0, t)=0, k_{x}(L, t)=k^{\beta}(L, t), t \in(0, T), \\
k(x, 0)=k_{0}(x), x \in[0, L],
\end{array}\right.
$$

where $r \geq 2, \alpha, \beta, L>0$ and $T \in(0, \infty)$. Let $k_{0}(x)$ be a positive initial function satisfying the compatibility conditions for (1.1) and (1.2).

Email address: bselcuk@karabuk.edu.tr

Received: 02.12.2019; Accepted: 15.09.2020 
The solution of (1.1) and (1.2) blow up if

$$
\lim _{t \rightarrow T_{\beta}^{-}} \sup k(x, t) \rightarrow \infty,
$$

where $T_{\beta}$ is called the blow up time. Here, we will discuss blow up properties in (1.1) and (1.2) with the help of two following blow up problems. In [7], Ozalp and Selcuk consider the problem

$$
\left\{\begin{array}{l}
u_{t}=u_{x x}, 0<x<L, 0<t<T, \\
u_{x}(0, t)=u^{\alpha}(0, t), u_{x}(L, t)=u^{\beta}(L, t), 0<t<T, \\
u(x, 0)=u_{0}(x), 0 \leq x \leq L,
\end{array}\right.
$$

where $\alpha, \beta$ are positive constants and $T \leq \infty$ and the initial function $u_{0}(x)$ is a nonnegative smooth function. They denote steady state of $u$ by $U$. They show that $u$ exists globally if $\alpha>\beta$ and $u_{0} \leq U(0)$ and $u$ blows up in a finite time if $\beta \geq \alpha, \beta>1$ and $u_{0}^{\prime \prime}(x) \geq$ 0 in $(0, L)$. In [5], Lin and Wang consider the problem

$$
\left\{\begin{array}{l}
u_{t}=u_{x x}+u^{\alpha}(0, t), 0<x<1,0<t<T, \\
u_{x}(0, t)=0, u_{x}(L, t)=u^{\beta}(1, t), 0<t<T, \\
u(x, 0)=u_{0}(x), 0 \leq x \leq 1,
\end{array}\right.
$$

where $\alpha, \beta$ are positive constants and $T \leq \infty$ and the initial function $u_{0}(x)$ is a nonnegative smooth function. They show that $u$ exists globally if $\max (\alpha, \beta) \leq 1$ and $u$ blows up in a finite time if $\max (\alpha, \beta)>1$ and $u_{0}^{\prime}(x) \geq 0$ in $(0,1)$. In [2], Fu et al. consider the same problem. Under certain conditions, they prove that the blow up point occurs only at the boundary. They derive the time asymptotic of solutions near the blow up time with the help of Giga-Kohn transform ([3]). Further, they prove that the blow up is complete.

So far in literature, the parabolic problem for non-Newtonian equations with two nonlinear sources have been studied for a few. Motivated by the problem [5] and [7], we discuss these situation in the present paper.

In Section 2, we treat (1.1) where we give an existence result and a blow up criterion. We show that finite time blow up occurs, and $x=L$ is a single blow up point under specific conditions. Further, we obtain a blow up rate and an estimate for the blow up time. In section 3, we give an existence result for (1.2). We show that finite time blow up occurs and $x=L$ is a single blow up point under specific conditions. Finally, we obtain blow up rates and estimates for the blow up time.

\section{Blow up of (1.1)}

In this section, we discuss blow up properties of (1.1). We assume that $k_{0}^{\prime}(x) \geq$ 0 and $k_{0}^{\prime \prime}(x) \geq 0$ in $(0, L)$. These assumptions guarantee that blow up will occur in finite time.

Remark 2.1. It is easily prove that, if $k_{0}^{\prime}(x) \geq 0$ and $k_{0}^{\prime \prime}(x) \geq 0$ in $(0, L)$ then $k_{x}, k_{t}>0$ in $[0, L] \times(0, T)$ by maximum principles.

Now, we rewrite the problem (1.1) into the following form:

$$
\left\{\begin{array}{l}
k_{t}=S(k) k_{x x}, 0<x<L, 0<t<T, \\
k_{x}(0, t)=k^{\alpha}(0, t), k_{x}(L, t)=k^{\beta}(L, t), 0<t<T, \\
k(x, 0)=k_{0}(x), 0 \leq x \leq L,
\end{array}\right.
$$

where $S(k)=(r-1) k_{x}^{r-2}$ and $k_{x}(x, t)>0$ in $(0, L) \times(0, T)$. Instead of $(1.1)$, we use (2.1) in this section for convenience. 
Definition 2.2. [7] $\mu$ is called an upper solution of the problem (1.3) if $\mu$ satisfies the following conditions:

$$
\begin{aligned}
& \mu_{t}-\mu_{x x} \geq 0,0<x<L, 0<t<T, \\
& \mu_{x}(0, t) \leq \mu^{\alpha}(0, t), \mu_{x}(L, t) \geq \mu^{\beta}(L, t), 0<t<T, \\
& \mu(x, 0) \geq u_{0}(x), 0 \leq x \leq L .
\end{aligned}
$$

It is a lower solution when the inequalities are reversed.

Lemma 2.3. [7] Let $u\left(x, t, u_{0}\right)$ and $p\left(x, t, p_{0}\right)$ be solutions of the problem (1.3) with initial data given by $u_{0}(x)$ and $p_{0}(x)$, respectively. If $u_{0} \leq p_{0}$ then $u\left(x, t, u_{0}\right) \leq p\left(x, t, p_{0}\right)$ on $[0, L] \times[0, T)$.

Corollary 2.4. $\quad$ (i) If we select $S(k) \geq 1$ i.e. $k_{x} \geq(r-1)^{-1 /(r-2)}$ and $k_{0}(x) \leq$ $u_{0}(x)$, then the solution $k$ of (1.1) is a lower solution of the problem (1.2) from Definition 2.2 and Lemma 2.3. So, we obtain that $k$ exists globally since $\alpha>\beta$, $u_{0} \leq U(0)$ in $(1.2), k_{0}(x) \leq u_{0}(x)$ and $S(k) \geq 1$, i.e. $k_{x} \geq(r-1)^{-1 /(r-2)}$ in (1.1).

(ii) Similarly, if we select $S(k) \leq 1$ i.e. $k_{x} \leq(r-1)^{-1 /(r-2)}$ and $u_{0}(x) \leq k_{0}(x)$ then the solution $k$ of (1.1) is an upper solution of the the problem (1.2) from Definition 2.2 and Lemma 2.3. So, we obtain that $k$ blows up in a finite time since $u_{0}^{\prime \prime}(x) \geq 0$ in $(0, L), \beta \geq \alpha, \beta>1$ in $(1.2), u_{0}(x) \leq k_{0}(x)$ and $S(k) \leq 1$ i.e. $k_{x} \leq(r-$ $1)^{-1 /(r-2)}$ in (1.1).

Theorem 2.5. If $\beta>1, k_{0}^{\prime}(x) \geq 0$ in $(0, L)$ are satisfied, then the single blow up point is $x=L$.

Proof. Let $d_{1} \in[0, L), d_{2} \in\left(d_{1}, L\right], \sigma \in(0, T)$ and $\xi>0$. Define an auxiliary function

$$
\Delta(x, t)=k_{x}-\xi\left(x-d_{1}\right) k^{\beta} \text { in }\left[d_{1}, d_{2}\right] \times[\sigma, T) .
$$

Hence, $\Delta(x, t)$ satisfies

$$
\begin{aligned}
& \Delta_{t}-(r-1) k_{x}^{r-2} \Delta_{x x}= \\
& \quad(r-1)\left((r-2) k_{x}^{r-3} k_{x x}^{2}+2 \xi \beta k^{\beta-1} k_{x}^{r-1}+\xi \beta(\beta-1)\left(x-d_{1}\right) k^{\beta-2} k_{x}^{r}\right)>0,
\end{aligned}
$$

in $\left(d_{1}, d_{2}\right) \times[0, T)$ where $\beta>1, r \geq 2$ and $k_{x}>0$ which is obtained from Remark 2.1. Similarly, $\Delta(x, \sigma) \geq 0$ for $k_{x}>0$ and for sufficient small $\varepsilon$. Also

$$
\begin{aligned}
& \Delta\left(d_{1}, t\right)=k_{x}\left(d_{1}, t\right)>0, \\
& \Delta\left(d_{2}, t\right)=k_{x}\left(d_{2}, t\right)-\xi\left(d_{2}-d_{1}\right) k^{\beta}>0,
\end{aligned}
$$

for $t \in(\sigma, T)$. So, it is easily seen that $\Delta(x, t) \geq 0$ for $(x, t) \in\left[d_{1}, d_{2}\right] \times[0, T)$ by the maximum principle. That is, $k_{x} \geq \xi\left(x-d_{1}\right) k^{\beta}$ for $(x, t) \in\left[d_{1}, d_{2}\right] \times[\sigma, T)$. Hence, taking the integral for $x$ from $d_{1}$ to $d_{2}$, we have

$$
k\left(d_{1}, t\right) \leq\left[\frac{\xi(\beta-1)\left(d_{2}-d_{1}\right)^{2}}{2}\right]^{\frac{1}{-\beta+1}}<\infty .
$$

So $k$ does not blow up in $[0, L)$.

Theorem 2.6. If $\beta>1, k_{0}^{\prime}(x) \geq 0$ and $k_{x}(x, 0) \geq x k^{\beta}(x, 0)$ in $(0, L)$ are satisfied, then the single blow up point is $x=L$ and $L \leq 1$.

Proof. Define an auxiliary function

$$
\Delta(x, t)=k_{x}-x k^{\beta} \text { in }[0, L] \times[0, T) .
$$

Thus, $\Delta(x, t)$ satisfies

$$
\Delta_{t}-(r-1) k_{x}^{r-2} \Delta_{x x}=(r-1)\left((r-2) k_{x}^{r-3} k_{x x}^{2}+2 \beta k^{\beta-1} k_{x}^{r-1}+\beta(\beta-1) x k^{\beta-2} k_{x}^{r}\right) \geq 0,
$$


in $(0, L) \times(0, T)$, where $k_{x}>0$ and $\beta, r>1$. Besides, $\Delta(x, 0) \geq 0$ from $k_{x}(x, 0) \geq$ $x k^{\beta}(x, 0)$ in $(0, L)$ and

$$
\begin{aligned}
\Delta(0, t) & =k^{\alpha}(0, t)>0, \\
\Delta(L, t) & =(1-L) k^{\beta}(L, t) \geq 0,
\end{aligned}
$$

since $L \leq 1$ and $t \in(0, T)$. So, it is easily seen that $\Delta(x, t) \geq 0$ by the maximum principle, namely $k_{x} \geq x k^{\beta}$ for $(x, t) \in[0, L] \times[0, T)$. Hence, taking the integral for $x$ from $x$ to $L$, we have

$$
k(x, t) \leq\left[(\beta-1) \frac{L^{2}-x^{2}}{2}\right]^{\frac{1}{-\beta+1}}<\infty .
$$

So $k$ does not blow up in $[0, L)$.

In the rest of this section, we assume that

$$
k_{t}(L, t)=(r-1) k_{x}^{r-2}(L, t) k_{x x}(L, t), 0<t<T .
$$

Theorem 2.7. If $\beta>1, k_{0}^{\prime \prime}(x) \geq 0, k_{0}^{\prime}(x) \geq 0$ and $k_{x}(x, 0) \geq \frac{x}{L} x k^{\beta}(x, 0)$ in $(0, L)$, then there exist a positive constant $C$ such that

$$
k(L, t) \geq C(T-t)^{1 /(2-\beta r)},
$$

for $t$ sufficiently close to $T$.

Proof. Define an auxiliary function

$$
\Delta(x, t)=k_{x}(x, t)-\frac{x}{L} k^{\beta}(x, t) \text { in }[0, L] \times[0, T) .
$$

Thus, $\Delta(x, t)$ implies

$\Delta_{t}-(r-1) k_{x}^{r-2} \Delta_{x x}=(r-1)\left((r-2) k_{x}^{r-3} k_{x x}^{2}+\frac{2}{L} \beta k^{\beta-1} k_{x}^{r-1}+\frac{x}{L} \beta(\beta-1) k^{\beta-1} k_{x}^{r}\right)>0$, in $(0, L) \times(0, T)$, where $k_{x}>0$ and $\beta, r>1$. Besides, $\Delta(x, 0) \geq 0$ from $k_{x}(x, 0) \geq$ $\frac{x}{L} k_{0}^{\beta}(x)$ in $(0, L)$ and

$$
\begin{aligned}
\Delta(0, t) & =k^{\alpha}(0, t)>0, \\
\Delta(L, t) & =0,
\end{aligned}
$$

for $t \in(0, T)$. Thus, by the maximum principle, we can see that $\Delta(x, t) \geq 0$; that is

$$
k_{x}(x, t) \geq \frac{x}{L} k^{\beta}(x, t) .
$$

Therefore,

$$
\Delta_{x}(L, t)=\lim _{s \rightarrow 0^{+}} \frac{\Delta(L, t)-\Delta(L-s, t)}{s}=\lim _{s \rightarrow 0^{+}} \frac{-\Delta(L-s, t)}{s} \leq 0 .
$$

We have

$$
\Delta_{x}(L, t)=k_{x x}-\frac{1}{L} k^{\beta}-\beta k^{2 \beta-1} \leq 0 .
$$

Consequently, using the assumption (2.2),

$$
k_{x x}(L, t) \leq\left(\frac{1+\beta L}{L}\right) k^{2 \beta-1},
$$

and we obtain

$$
k_{t}(L, t) \leq\left(\frac{(1+\beta L)(r-1)}{L}\right) k^{\beta r-1} .
$$

Taking the integral for $t$ from $t$ to $T$, we have a blow up rate

$$
k(L, t) \geq C(T-t)^{1 /(2-\beta r)},
$$


where $C=\left[\left(\frac{(1+\beta L)(r-1)}{L}\right)(\beta r-2)\right]^{1 /(2-\beta r)}$. In addition, we have an upper estimate $k_{0}^{2-\beta r}(L) /(\beta r-2)$ of blow up time.

\section{Blow up of (1.2)}

In this section, we discuss blow up properties of (1.2). We assume that $k_{0}^{\prime}(x) \geq$ 0 and $\left(\left|k_{0}^{\prime}(x)\right|^{r-2} k_{0}^{\prime}(x)\right)_{x}+k_{0}^{\alpha}(x) \geq 0$ in $(0, L)$. These assumptions guarantee that blow up will occur in finite time.

Remark 3.1. It is easily proved that, if $k_{0}^{\prime}(x) \geq 0$ and $\left(\left|k_{0}^{\prime}(x)\right|^{r-2} k_{0}^{\prime}(x)\right)_{x}+k_{0}^{\alpha}(x) \geq 0$ in $(0, L)$, then $k_{x}, k_{t}>0$ in $[0, L] \times(0, T)$ by the maximum principles.

Now, we rewrite the problem (1.2) into the following form

$$
\left\{\begin{array}{l}
k_{t}=S(k) k_{x x}+k^{\alpha}, 0<x<L, 0<t<T, \\
k_{x}(0, t)=0, k_{x}(L, t)=k^{\beta}(L, t), 0<t<T, \\
k(x, 0)=k_{0}(x), 0 \leq x \leq L,
\end{array}\right.
$$

where $k_{x}(x, t)>0$ in $(0, L) \times(0, T), S(k)=(r-1) k_{x}^{r-2}$. Instead of (1.2), we use (3.1) in this section for convenience.

Definition 3.2. $\lambda$ is called a lower solution of problem (1.4) if $\lambda$ satisfies the following conditions:

$$
\begin{aligned}
& \lambda_{t}-\lambda_{x x} \leq \lambda^{\alpha}, 0<x<L, 0<t<T \\
& \lambda_{x}(0, t)=0, \lambda_{x}(1, L) \leq \lambda^{\beta}(L, t), 0<t<T, \\
& \lambda(x, 0) \leq u_{0}(x), 0 \leq x \leq L .
\end{aligned}
$$

It is an upper solution when the inequalities are reversed.

Lemma 3.3. Let $u$ be a solution and $\mu$ be an upper solution of problem (1.4) in $[0, L] \times$ $[0, T)$. Then $\mu \geq u$ in $[0,1] \times[0, T)($ See Lemma 2.4 in $[5])$.

Corollary 3.4. If we select $S(k) \geq 1$ i.e. $k_{x} \geq(r-1)^{-1 /(r-2)}$ and $k_{0}(x) \leq u_{0}(x)$, solution $k$ of (1.2) is a lower solution of the problem (1.4) from Definition 3.2 and Lemma 3.3. Then, $k$ exists globally since $\max (\alpha, \beta) \leq 1$ in (1.4), $S(k) \geq 1$ i.e. $k_{x} \geq(r-1)^{-1 /(r-2)}$ in (1.2) and $k_{0}(x) \leq u_{0}(x)$.

Theorem 3.5. A solution $k$ of (1.2) blows up since $\max (\alpha, \beta(r-1))>1$ and $k_{0}^{\prime}(x) \geq 0$ in $(0, L)$. (See Theorem 2.1 in [5])

Proof. Suppose $\max (\alpha, \beta(r-1))>1$ and $k_{0}^{\prime}(x) \geq 0$ in $[0, L]$ hold. Define an auxiliary function; $M(t)=\int_{0}^{L} k(x, t) d x, 0<t<T$. Then we have,

$$
M^{\prime}(t)=k^{\beta(r-1)}(L, t)+\int_{0}^{L} k^{\alpha}(x, t) d x \geq L^{-\beta(r-1)} M^{\beta(r-1)}+\int_{0}^{L} k^{\alpha}(x, t) d x,
$$

since $k_{x}>0$. If $\beta(r-1)>1$, then $M(t)$ must blow up in a finite time, that is $k(x, t)$ must blow up in a finite time. Also, we have

$$
M^{\prime}(t)=k^{\beta(r-1)}(L, t)+\int_{0}^{L} k^{\alpha}(x, t) d x \geq k^{\beta(r-1)}(L, t)+C M^{\alpha}(t),
$$

with the help of the Hölder's inequality. If $\alpha>1$, then $M(t)$ blows up in a finite time, that is $k(x, t)$ must blow up in a finite time.

Theorem 3.6. If $\alpha>1$ (or $\beta(r-1)>1), k_{0}^{\prime}(x) \geq 0$ in $(0, L)$ are satisfies, then the single blow up point is $x=L$. 
Proof. Let $d_{1} \in[0, L), d_{2} \in\left(d_{1}, L\right], \sigma \in(0, T)$ and $\xi>0$. Define the auxiliary function

$$
\Delta(x, t)=k_{x}-\xi\left(x-d_{1}\right) k^{\alpha} \text { in }\left[d_{1}, d_{2}\right] \times[\sigma, T) .
$$

Hence, $\Delta(x, t)$ satisfies

$$
\begin{aligned}
& \Delta_{t}-(r-1) k_{x}^{r-2} \Delta_{x x}-\alpha k^{\alpha-1} \Delta= \\
& \quad(r-1)\left((r-2) k_{x}^{r-3} k_{x x}^{2}+2 \xi \alpha k^{\alpha-1} k_{x}^{r-1}+\xi \alpha(\alpha-1)\left(x-d_{1}\right) k^{\alpha-2} k_{x}^{r}\right)>0,
\end{aligned}
$$

in $\left(d_{1}, d_{2}\right) \times[0, T)$, where $\alpha>1, r \geq 2$ and $k_{x}>0$ which is resulted from Remark 3.1. Similarly, $\Delta(x, \sigma) \geq 0$ from $k_{x}>0$, if $\varepsilon$ is sufficient small. Also

$$
\begin{aligned}
& \Delta\left(d_{1}, t\right)=k_{x}\left(d_{1}, t\right)>0, \\
& \Delta\left(d_{2}, t\right)=k_{x}\left(d_{2}, t\right)-\xi\left(d_{2}-d_{1}\right) k^{\alpha}>0,
\end{aligned}
$$

for $t \in(\sigma, T)$. So, it is easily seen that $\Delta(x, t) \geq 0$ for $(x, t) \in\left[d_{1}, d_{2}\right] \times[0, T)$ by the maximum principle. That is, $k_{x} \geq \xi\left(x-d_{1}\right) k^{\alpha}$ for $(x, t) \in\left[d_{1}, d_{2}\right] \times[\sigma, T)$. Hence, taking the integral for $x$ from $d_{1}$ to $d_{2}$, we have

$$
k\left(d_{1}, t\right) \leq\left[\frac{\xi(\alpha-1)\left(d_{2}-d_{1}\right)^{2}}{2}\right]^{\frac{1}{-\alpha+1}}<\infty .
$$

So $k$ does not blow up in $[0, L)$.

If we follow similar process as above and define $\Delta(x, t)=k_{x}-\xi\left(x-d_{1}\right) k^{\beta(r-1)}$ in $\left[d_{1}, d_{2}\right] \times[\sigma, T)$, we get

$$
k\left(d_{1}, t\right) \leq\left[\frac{\xi(\beta(r-1)-1)\left(d_{2}-d_{1}\right)^{2}}{2}\right]^{\frac{1}{-\beta(r-1)+1}}<\infty .
$$

So $k$ does not blow up in $[0, L)$.

In the rest of this section, we assume that

$$
k_{t}(L, t)=(r-1) k_{x}^{r-2}(L, t) k_{x x}(L, t)+k^{\alpha}(L, t), 0<t<T .
$$

Theorem 3.7. If $\beta>1, k_{0}^{\prime}(x) \geq 0,\left(\left|k_{0}^{\prime}(x)\right|^{r-2} k_{0}^{\prime}(x)\right)_{x}+k_{0}^{\alpha}(x) \geq 0$ and $k_{x}(x, 0) \geq$ $\frac{x}{L} x k^{\beta}(x, 0)$ in $(0, L)$ are satisfied, then there exist positive constants $C_{2}$ and $C_{3}$ such that

$$
\begin{aligned}
& k(L, t) \geq C_{2}(T-t)^{1 /(2-\beta r)}, \quad \text { if } \alpha<\beta r-1, \\
& k(L, t) \geq C_{3}(T-t)^{1 /(1-\alpha)}, \quad \text { if } \alpha \geq \beta r-1,
\end{aligned}
$$

for $t$ sufficiently close to $T$.

Proof. Define an auxiliary function

$$
\Delta(x, t)=k_{x}(x, t)-\frac{x}{L} k^{\beta}(x, t) \text { in }[0, L] \times[0, T) .
$$

Thus, $\Delta(x, t)$ implies

$$
\begin{aligned}
& \Delta_{t}-(r-1) k_{x}^{r-2} \Delta_{x x}-\alpha k^{\alpha-1} \Delta= \\
& \quad(r-1)\left((r-2) k_{x}^{r-3} k_{x x}^{2}+\frac{2}{L} \beta k^{\beta-1} k_{x}^{r-1}+\frac{x}{L} \beta(\beta-1) k^{\beta-1} k_{x}^{r}\right)>0,
\end{aligned}
$$

in $(0, L) \times(0, T)$, where $\beta>1, r \geq 2$ and $k_{x}>0$. Besides, $\Delta(x, 0) \geq 0$ from $k_{x}(x, 0) \geq$ $\frac{x}{L} k_{0}^{\beta}(x)$ in $(0, L)$ and

$$
\begin{aligned}
\Delta(0, t) & =k^{\alpha}(0, t)>0, \\
\Delta(L, t) & =0,
\end{aligned}
$$


for $t \in(0, T)$. Thus, by the maximum principle, we can see that $\Delta(x, t) \geq 0$; that is

$$
k_{x}(x, t) \geq \frac{x}{L} k^{\beta}(x, t) .
$$

Therefore

$$
\Delta_{x}(L, t)=\lim _{s \rightarrow 0^{+}} \frac{\Delta(L, t)-\Delta(L-s, t)}{s}=\lim _{s \rightarrow 0^{+}} \frac{-\Delta(L-s, t)}{s} \leq 0 .
$$

We have

$$
\Delta_{x}(L, t)=k_{x x}-\frac{1}{L} k^{\beta}-\beta k^{2 \beta-1} \leq 0 .
$$

Hence, using the assumption (3.2)

$$
k_{x x}(L, t) \leq\left(\frac{1+\beta L}{L}\right) k^{2 \beta-1}
$$

and we have

$$
k_{t}(L, t) \leq\left(\frac{(1+\beta L)(r-1)}{L}\right) k^{\beta r-1}+k^{\alpha} .
$$

Taking the integral for $t$ from $t$ to $T$, we have blow up rates

$$
\begin{aligned}
& k(L, t) \geq C_{2}(T-t)^{1 /(2-\beta r)}, \quad \text { if } \alpha<\beta r-1, \\
& k(L, t) \geq C_{3}(T-t)^{1 /(1-\alpha)}, \quad \text { if } \alpha \geq \beta r-1,
\end{aligned}
$$

where

and

$$
C_{2}=\left[\left(\frac{(1+\beta L)(r-1)+L}{L}\right)(\beta r-2)\right]^{1 /(2-\beta r)}
$$

$$
C_{3}=\left[\left(\frac{(1+\beta L)(r-1)+L}{L}\right)(\alpha-1)\right]^{1 /(1-\alpha)},
$$

respectively. In addition, we have upper estimates

$$
\begin{gathered}
k_{0}^{2-\beta r}(L) /(\beta r-2), \text { if } \alpha<\beta r-1, \\
k_{0}^{1-\alpha}(L) /(\alpha-1), \text { if } \alpha \geq \beta r-1,
\end{gathered}
$$

of blow up time.

\section{Conclusions}

The main results in (1.1) are the following;

(i) the single blow up point is $x=L$ since $\beta>1$ and $k_{0}^{\prime}(x) \geq 0$ in $(0, L)$,

(ii) the single blow up point is $x=L$ and $L \leq 1$ since $\beta>1, k_{0}^{\prime}(x) \geq 0, k_{0}^{\prime \prime}(x) \geq$ 0 and $k_{x}(x, 0) \geq x k^{\beta}(x, 0)$ in $(0, L)$,

(iii) the lower bound of blow up rate is $k(L, t) \geq C(T-t)^{1 /(2-\beta r)}$ since $\beta>1, k_{0}^{\prime}(x) \geq 0$, $k_{0}^{\prime \prime}(x) \geq 0$ and $k_{x}(x, 0) \geq \frac{x}{L} x k^{\beta}(x, 0)$ in $(0, L)$.

The main results in (1.2) are the following;

(i) the single blow up point is $x=L$ since $\max (\alpha, \beta(r-1))>1$ and $k_{0}^{\prime}(x) \geq 0$ in $(0, L)$.

(ii) lower bounds of blow up rates are

$$
\begin{aligned}
& \text { if } \alpha<\beta r-1, k(L, t) \geq C_{2}(T-t)^{1 /(2-\beta r)}, \\
& \text { if } \alpha \geq \beta r-1, k(L, t) \geq C_{3}(T-t)^{1 /(1-\alpha)},
\end{aligned}
$$

where $k_{0}^{\prime}(x) \geq 0, k_{0}^{\prime \prime}(x) \geq 0$ and $k_{x}(x, 0) \geq \frac{x}{L} x k^{\beta}(x, 0)$ in $(0, L)$.

Acknowledgment. The author is very grateful to the referees for their valuable suggestions, which helped to improve the paper significantly. 


\section{References}

[1] Z. Cui, Critical curves of the non-Newtonian polytropic filtration equations coupled with nonlinear boundary conditions, Nonlinear Anal. 68 (10), 3201-3208, 2008.

[2] S.C. Fu, J.S. Guo and J-C. Tsai, Blow-up behavior for a semilinear heat equation with a nonlinear boundary condition, Tohoku Math. J. (2), 55 (4), 565-581, 2003.

[3] Y. Giga and R.V. Kohn, Asymptotic self-similar blowup of semilinear heat equations, Comm. Pure Appl. Math. 38, 297-319, 1985.

[4] Z. Liang, On the blow-up set for non-Newtonian equation with a nonlinear boundary condition, Abstr. Appl. Anal. 2010, 1-12, 2010.

[5] Z. Lin and M. Wang, The blow up properties of solutions to semilinear heat equation with nonlinear boundary conditions, Z. Angew. Math. Phys. 50, 361-374, 1999.

[6] E. Novruzov, On blow up of solution of nonhomogeneous polytropic equation with source, Nonlinear Anal. 71 (9), 3992-3998, 2009.

[7] N. Ozalp and B. Selcuk, Blow up and quenching for a problem with nonlinear boundary conditions, Electron. J. Differential Equations, 2015 (192), 1-11, 2015.

[8] Z. Wang, J. Yin and W.C. Wang, Critical exponents of the non-Newtonian polytropic filtration equation with nonlinear boundary condition, Appl. Math. Lett. 20, 142-147, 2007.

[9] Z.Yang and Q. Lu, Nonexistence of positive solutions to a quasilinear elliptic system and blow-up estimates for a non-Newtonian filtration system, Appl. Math. Lett. 16 (4), 581-587, 2003.

[10] H. Zhang, Z. Liu and W. Zhan, Growth estimates and blow-up in quasilinear parabolic problems, Appl. Anal. 86 (2), 261-268, 2007.

[11] J. Zhou, Global existence and blow-up of solutions for a Non-Newton polytropic filtration system with special volumetric moisture content, Comput. Math. Appl. 71 (5), 1163-1172, 2016. 